\title{
EL MODELO INTERACTIVO DE ESCUCHA Y LECTURA: UNA ESTRATEGIA PARA DISEÑAR ACTIVIDADES COMUNICATIVAS A PARTIR DE LAS DESTREZAS PASIVAS
}

Eva Schnell

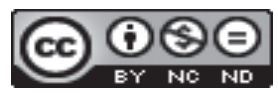

Esta obra está bajo una licencia Creative Commons Reconocimiento-No Comercial-Sin Obra Derivada 



\title{
EL MODELO INTERACTIVO DE ESCUCHA Y LECTURA: UNA ESTRATEGIA PARA DISEÑAR ACTIVIDADES COMUNICATIVAS A PARTIR DE LAS DESTREZAS PASIVAS

\author{
THE LISTENING AND READING INTERACTIVE MODEL: A \\ STRATEGY TO DESIGN COMMNICATIVE ACTIVITIES THROUGH \\ PASSIVE SKILLS
}

Eva Schnell

\begin{abstract}
RESUMEN
El presente artículo propone incorporar el Modelo Interactivo de Escucha y Lectura, según la perspectiva de Swaffar, Arens y Byrnes (1991), como una estrategia e hilo conductor para desarrollar, organizar y escalonar las actividades de una unidad didáctica o plan de clase. Esto con la intención de destacar el Modelo como un recurso integrador que provee oportunidades para seleccionar y organizar un repertorio motivante con actividades interactivas, significativas y contextualizadas a partir del área de la comprensión (auditiva y lectora), sin dejar de incluir las actividades del área de la expresión (oral y escrita) y aspectos cognitivos para derivar significado, recrear y reaccionar ante el texto a partir de posiciones personales.

Palabras clave: destrezas pasivas, actividades interactivas, estrategias metodológicas, lectura interactiva, comprensión auditiva.
\end{abstract}

\begin{abstract}
This article is intended to incorporate the Interactive Listening and Reading Model, from the perspective of Swaffar, Arens and Byrnes (1991) as a strategy and thread to develop, organize and scale up the activities of a teaching unit or lesson plan. This with the intention of highlighting the Model as an integrative tool providing opportunities to select and organize a motivating repertoire with interactive, meaningful and contextualized activities starting from the area of comprehension (listening and reading), including also expressive activities (oral and written) as well as cognitive aspects to generate meaning, recreation and reaction to the text from a personal perspective.

Key words: passive skills, interactive activities, methodological strategies, interactive reading, listening.
\end{abstract}

\section{Modelos de procesamiento de lectura y la enseñanza de segundas lenguas}

Samuels y Kamil (1993), reseñan a grandes trazos la historia de los modelos de procesamiento de lectura. Señalan como punto de partida el desarrollo de las investigaciones

\footnotetext{
M. L. Eva Schnell. Universidad Estatal a Distancia. Profesora del Centro de Idiomas. Costa Rica. Correo electrónico: eva.traducom@gmail.com
} 
sobre el movimiento de los ojos durante la lectura publicadas por Emile Javal en 1879. Comentan que los primeros trabajos relacionados con el procesamiento de la lectura proponen un enfoque unidireccional sintético, lo que implica comenzar el proceso de lectura decodificando las palabras del texto (pronunciación y morfología, por ejemplo). Culminan la revisión con la propuesta derivada de las perspectivas psicolingüísticas de Goodman (1993) sobre la lectura y la relacionan con un enfoque analítico. Esta propuesta de procesamiento de lectura sostiene la conveniencia de comenzar el proceso de comprensión por los aspectos más generales del texto relacionados con las experiencias previas del lector.

La perspectiva de Goodman (1993) define la lectura como un proceso receptivo y psicolingüístico que empieza con una representación lingüística superficial codificada por el escritor y que termina con el sentido que el lector construye.

Eskey (1993) reconoce que el punto de vista que sostiene la perspectiva de que la lectura relacionada con el procesamiento unidireccional, analítico y decodificador ha renovado la perspectiva de los profesores de segundas lenguas. También reconoce que les ha ayudado a entender mejor a sus estudiantes durante las actividades de lectura y a seleccionar los materiales y estrategias para sus clases. Sin embargo, señala que los modelos analíticos de procesamiento de lectura tienen sus limitaciones ya que tienden a enfatizar el uso de destrezas complejas para activar una aproximación descendente. A este respecto, Samuels y Kamil (1993) señalan que si la experiencia previa del lector no está relacionada con el tema de la lectura, este tendrá dificultades para generar predicciones. Por otro lado, Swaffar, Arens y Byrnes (1991), señalan que el modelo sintético de procesamiento de lectura que conceptualiza la lectura a partir del reconocimiento de las letras y palabras en un texto no es recomendable ni siquiera en los niveles iniciales de instrucción. Reconocen que puede ser útil cuando el estudiante tiene una información de entrada limitada fuera de la clase pero a la vez señalan que las investigaciones evidencian que solo el reconocimiento de las palabras en un texto no significa su comprensión.

Las insuficiencias de estos dos tipos de procesamiento de lectura, cuando son aplicados individualmente, generan observaciones de varios investigadores, entre ellos Eskey y Grabe (1993), quienes recomiendan que las dos estrategias, analítica y sintética, deben ser desarrolladas en forma conjunta ya que contribuyen con la comprensión exitosa del texto. A ese respecto, Carrel (1993) también apoya la aplicación de un modelo de procesamiento de lectura bidireccional (analítica y sintética) y recomienda que las actividades estén organizadas de manera que propicien la interacción de los dos modelos.

\section{El Modelo Interactivo de Escucha y Lectura y la enseñanza de segundas lenguas}

El Modelo Interactivo de Escucha y Lectura se caracteriza justamente por involucrar en su propuesta la interacción de los dos modelos de procesamiento de lectura (analítica y sintética). Propone una cronología escalonada de actividades, comprendida por seis etapas que estimulan la búsqueda y utilización no solo de estrategias facilitadoras de la comprensión sino también de la expresión oral y escrita y las diferentes modalidades relacionadas con cada una de las destrezas.

Promueve la interacción del texto con el lector u oyente ya que este es llevado en cada una de las etapas a aplicar las estrategias del enfoque sintético y del enfoque analítico. Además, de acuerdo con Shrum y Glisan (1994), el modelo es integrador, ya que provee oportunidades 
para combinar actividades orales, de escucha, lectura, escritura, así como aspectos cognitivos para derivar significado, recrear y reaccionar ante el texto a partir de posiciones personales por lo que puede ser aplicado con textos orales, escritos, videos y adecuarse a propuestas con textos literarios, cuentos cortos, obras de teatro y poemas.

\section{Etapas del Modelo}

Es posible alternar los textos de comprensión (audio o lectura), seleccionar diferentes áreas temáticas y motivaciones para cada clase. También, es importante organizar el orden de las destrezas en el diseño de la clase o unidad didáctica, todo según los objetivos propuestos. Cada clase no necesariamente tiene que cumplir con las seis etapas. El siguiente desglose de las etapas del Modelo Interactivo es una adaptación de Shrum y Glisan (1994) y enriquecida, principalmente, con los aportes de las lecturas de, Swaffar, Arens y Byrnes (1991), Cassany, Luna y Sanz (2008), Lucchi-Riester (2007), Richards (1990) y Eskey (1993).

\subsection{Etapa I - Pre escucha o lectura (preparación)}

Involucra al grupo en el tema que se va a proponer para que se sitúe en el texto antes de enfrentarse a él. Esto con la intención de acercar el estudiante no solo al léxico básico de la temática sino para activar su esquema cultural o experiencias anteriores. En esta etapa el estudiante está activo para predecir o suponer lo que encontrará u ocurrirá en la lectura o audio (cómo seguirá, cómo terminará, por ejemplo) haciendo uso de las pistas que las estructuras gramaticales, el contexto y el léxico le brindan. Como resumen, se puede decir que en esta etapa el estudiante organiza la información de entrada según su experiencia previa. Esta experiencia previa está relacionada con la cultura, un espacio ideológico que funciona como una memoria y referencia (Cros, 1997, p. 9). Cuando la pre lectura involucra aspectos culturales, es necesario que los profesores intenten conducir a sus alumnos hacia una mayor empatía intercultural.

Relación con el significado del texto - El primer acercamiento les permitirá hacer predicciones e interactuar con la información, ideas y conceptos nuevos, que solo tendrán significado si se pueden relacionar con algo que ya conocen. La relación entre la información nueva y la ya existente ayuda el estudiante a encontrar el sentido del texto con más rapidez. La comprensión del texto se alcanza a partir de la interrelación entre lo que el lector lee y lo que ya sabe sobre el tema.

Relación con el léxico - La contextualización previa contribuye con la selección de los esquemas necesarios para acercarse al texto. Para ello, los participantes deben compartir el mismo significado referencial para el léxico empleado ya que usan palabras y frases como pistas para llegar al significado. La conexión entre la forma y el significado favorece la transformación de la información de entrada en información de salida.

Actividades - Los estudiantes pueden realizar una lectura globalizada, rápida y general para captar la esencia del texto. Observan el formato, el diseño gráfico (tipografía, presentación, negritas, cursivas, siglas, etc.), títulos, subtítulos y apartados, por ejemplo. No examinan la sintaxis ni la puntuación. Pueden también definir el objetivo del texto buscando datos específicos con una lectura focalizada, más lenta.

En esta etapa, discutir el título de un texto podría ser interesante ya que este es un elemento contextualizador. 


\subsection{Etapa II - Identificar elementos principales (comprensión)}

Relación con el significado del texto - En el segundo paso, el estudiante explora el texto para obtener más información que lo acerque a la idea principal.

Relación con el lenguaje del texto - Los estudiantes reconocerán los elementos discursivos (estructuras gramaticales, cláusulas y el orden de las palabras, por ejemplo).

Actividades - El profesor selecciona las actividades que permitan discutir y explorar la identificación de las ideas principales según los objetivos de la clase. Además, explora el vocabulario o las estructuras que cambien el significado del texto. El profesor puede proponer, por ejemplo, una lista de palabras relacionadas con la lectura o el audio y sugerir una búsqueda en el diccionario. Otro ejemplo puede ser un juego de pareo en el cual los estudiantes compaginen la información de la etapa anterior con imágenes o bien las posibles ilustraciones de la etapa anterior con la lista de palabras de esta etapa. Lo importante, como ya se ha mencionado, es escalonar la aproximación del estudiante al texto y organizar el orden de las destrezas según los objetivos de la propuesta.

\subsection{Etapa III - Identificar detalles (interpretación)}

Relación con el significado del texto - En el tercer paso, la etapa medular del Modelo, el profesor propone la audición o lectura ya que en las dos etapas anteriores se prepararon para ello. Los estudiantes, durante la lectura o audición, relacionan los detalles con la idea principal.

Relación con el lenguaje del texto - Los estudiantes establecen conexiones entre la idea principal y los detalles.

Actividades - A partir de las ideas principales identificadas en la Etapa II, después de escuchar o leer, los estudiantes pueden, por ejemplo, aportar detalles por escrito. También es posible proponer que seleccionen, de una lista, algunos detalles compatibles y lo justifiquen por escrito para luego discutirlos en grupo; se puede proponer una actividad con el grupo para completar un cuadro en la pizarra, cada estudiante aporta un detalle y lo justifica o bien ofrece su opinión. También es válido, para los niveles iniciales la discriminación de sonidos: indicar en qué palabras se escucha un determinado sonido, diferenciar pares de palabras del tipo paja/caja, corro/coro, por ejemplo. Seguir instrucciones: hacer un dibujo, completar un mapa, ordenar objetos a partir de la información obtenida de la audición puede resultar interesante. Actividades de relación o distinción también son adecuadas para la Etapa III. Si los alumnos escuchan, por ejemplo, la descripción de una persona, una casa, una mascota, pueden elegir un dibujo que corresponda a la descripción que escucharon. Los estudiantes pueden centrar su atención en obtener información específica para completar cuadros o diagramas o bien participar de actividades de respuesta (correcta/incorrecta). Las canciones pueden ser escuchadas para completar espacios, ordenar frases, ordenar fragmentos desordenados o identificar estructuras, por ejemplo.

Específicamente para la comprensión lectora, Cassany et ál (2008) recomiendan las siguientes actividades:

a. Seguir instrucciones escritas o bien localizar una información concreta.

b. Ordenar dibujos de acuerdo con un texto, completar los espacios en blanco en un texto.

c. Emparejar dibujos y textos.

d. Imaginar el desenlace de una historia. 

e. Elaborar frases que resuman el contenido.
f. Poner título a un texto.
g. Resumir el texto con un número determinado de palabras.
h. Reconstruir textos que han sido cortados y mezclados.
i. Contestar a preguntas formuladas por el profesor.
j. Elaborar preguntas a respuestas dadas.

\subsection{Etapa IV - Organizar / revisar ideas principales y detalles (aplicación)} la Etapa III.

El estudiante, después de la lectura o audición, revisa la información reunida hasta

Relación con el significado del texto - En el cuarto paso el estudiante organiza y sistematiza la información por sí mismo, revisa la idea principal y, además, aporta detalles.

Relación con el lenguaje del texto - Los estudiantes usan el vocabulario del texto para mejorar sus resúmenes, apuntes o su participación en las intervenciones orales.

Actividades - Las actividades de interacción (en pares o grupos) comparan la información recopilada por cada uno para verificar si es consistente con la ofrecida por el texto o audio.

\subsection{Etapa V - Recrear el texto (reconstrucción)}

Los estudiantes reconstruyen la información.

Relación con el significado del texto - La recreación del texto, caracteriza la Etapa $\mathrm{V}$, en la cual el texto es un pretexto para desarrollar vocabulario relacionado con el tema, desarrollar la interculturalidad e integrar el contenido gramatical abordado.

Relación con el lenguaje del texto - Los estudiantes amplían las frases del texto o apuntes de vocabulario recopilados para transformarlos en oraciones y párrafos.

Actividades- Propuestas relacionadas con la destreza escrita son apropiadas para esta etapa ya que proponen la reconstrucción y resumen de la información recopilada. Una propuesta interactiva para pequeños grupos puede ser preparar un guión para una presentación (Lucchi-Riester, 2007), un programa de radio o un anuncio, por ejemplo. Como tarea, queda muy bien y se retoma en la clase siguiente, con la presentación oral, por ejemplo, del programa de radio.

\subsection{Etapa VI - Reaccionar ante el texto / Explorar intertextualidad (reacción)}

Relación con el significado del texto - El estudiante, en la Etapa VI, externa opiniones, reacciona ante el texto y lo relaciona con otros textos.

Relación con el lenguaje del texto - Los estudiantes usan el texto como apoyo para externar sus opiniones.

Actividades - Actividades de interacción oral son adecuadas para esta etapa ya que los estudiantes externan y comparten sus opiniones. Transforman las oraciones y párrafos de la etapa $\mathrm{V}$ en enunciados concretos y pertinentes.

\section{4. ¿Por qué a partir de destrezas pasivas o receptivas?}

A través del tiempo el desarrollo del contexto histórico posicionó la comunicación como un paradigma en la metodología del tratamiento pedagógico de las L2 por lo que "las 
destrezas de escucha y lectura han recibido menos atención que las destrezas productivas, como la oral y la escritura, y los profesores han asumido que las destrezas receptivas se desarrollarían por sí mismas" (Shrum y Glisan, 1994, p. 112). Es importante incorporar la posición de Swaffar, Arens y Byrnes (1991), quienes mencionan que si la lectura y escritura están ausentes en los niveles iniciales de enseñanza, los estudiantes tendrán dificultades para ajustarse a los trabajos que exigen esta destreza y eso repercute en el proceso de adquisición.

El Modelo, además, responde a la convergencia entre otros principios y propuestas teóricas relacionadas con la adquisición y enseñanza de segundas lenguas. Entre ellas encuentra eco en la Teoría del Filtro Afectivo que sostiene que las variables del dominio afectivo influyen en la adquisición. Krashen (1981, p. 32), al comentar las características de la Hipótesis del Filtro Afectivo menciona que "los objetivos pedagógicos no se limitan a ofrecer solo una información de entrada comprensible, sino también, crear un ambiente que promueva filtros afectivos relajados”. El Modelo, al propiciar la posibilidad para alternar no solamente las destrezas sino las especificidades de cada una, ofrece un abanico de oportunidades para que los estudiantes participen más activamente en las actividades y destrezas en las cuales se sientan más cómodos. Además, el andamiaje del Modelo permite que la información de entrada sea provista según las recomendaciones de Krashen (1981): a. comprensible; b. parcialmente superior al nivel efectivo de competencia (i+1), motivante; c. relevante, no presentada en secuencias gramaticales; $d$. recibida en un ambiente relajado que promueva una actitud positiva y, además, que desencadene una reflexión en el estudiante sin generar ansiedad y frustración, lo que ajustaría el filtro afectivo y en consecuencia inhibiría el acceso a la información de entrada.

Si bien es cierto que, como señala Krashen (1981, p. 59), "el discurso al cual el estudiante se expone en el aula es limitado sin importar lo natural que se pretenda presentarlo", también lo es el hecho de que el mundo real puede proveer más información de entrada, por lo que el objetivo de las clases no es sustituir la variedad que el estudiante encuentra fuera del salón de clases sino llevarlos al punto en que puedan aunar las experiencias del contacto con el mundo real con la información de entrada ofrecida en clase como subsidios para incrementar la competencia conversacional (Krashen, 1981). Esta posibilidad, por ejemplo, la provee la Etapa VI del Modelo - Reaccionar ante el texto.

Por otro lado, Shrum y Glisan (1994), mencionan que incluir el Modelo Interactivo de Escucha y Lectura en el diseño de unidades no solamente desarrolla las destrezas de comprensión sino que integra otras destrezas, lo que repercute en forma positiva en la adquisición. Schnell (2011) destacó el incremento de la faceta participativa y creativa en actividades de expresión escrita, generalmente no muy bienvenidas y relacionadas con actividades individuales, con una propuesta de expresión escrita extensiva en la modalidad de parejas (recrear un cuento clásico) para la Etapa V- Recrear el texto.

\section{Conclusiones}

La arquitectura del Modelo Interactivo, como elemento articulador, minimiza algunos de los principales retos que supone el diseño de las clases y unidades didácticas.

Antes de la lectura interactiva (Etapa III), etapa medular del Modelo, dos etapas preparatorias, relacionadas con otras destrezas y actividades, acercan el estudiante al texto, lo que evita la impresión de que, quizás por falta de tiempo, se llevó a la clase cualquier lectura ya que estructura claramente el desarrollo de la clase y estimulan la creatividad. 
Las clases fundamentadas en el Modelo Interactivo, como hilo conductor, siguen un plan ordenado que favorece retomarlas en un punto definido ante las eventuales interrupciones (preguntas y explicaciones, por ejemplo) sin afectar la continuidad de la gestión de clase. Además, las seis etapas constituyen una plataforma adecuada para alternar las destrezas según sus aspectos más específicos (expresión oral extensiva o expresión escrita de respuesta, por ejemplo) y enlazarlas con actividades compatibles (en grupo o individuales) y así generar transiciones fluidas. Además, estimula una participación activa sin que ello genere mucha tensión ya que la posibilidad de combinar las variables de cada destreza ofrece un abanico de oportunidades y alternativas para que los estudiantes participen más activamente en la combinación de actividades y destrezas en las cuales se sientan más cómodos. Como resultado los estudiantes mantendrán filtros afectivos bastante relajados aun durante las actividades más demandantes. No necesariamente en cada clase se deben agotar todas las seis etapas.

La presentación de la información de entrada de manera comprensible, contextualizada, organizada con textos auténticos y con temas afines a la experiencia previa de los estudiantes ofrece oportunidades para que generen predicciones (Etapa I) y expresen su propia opinión (Etapa VI) mediante la expresión oral y escrita en sus diversas variantes. Asimismo, a lo largo de la clase, las diferentes etapas del Modelo abren espacios para la reflexión ya que los estudiantes quedan expuestos a textos y actividades que pretenden un abordaje progresivo para alcanzar los objetivos propuestos.

\section{Ejemplo resumido de un plan de clase según el Modelo}

\section{Cuadro 1}

\begin{tabular}{|c|c|}
\hline Título & ¡Empezar el día! \\
\hline Estrategia $>$ & Modelo Interactivo - Etapas I y II \\
\hline Material $>$ & Folleto de actividades \\
\hline \multicolumn{2}{|c|}{$\begin{array}{l}\text { Objetivo general: Argumentar la intensidad de las preferencias, de las personas en general, } \\
\text { relacionadas con las rutinas de aseo durante el período de vacaciones. }\end{array}$} \\
\hline Estrategia & Actividades y destrezas \\
\hline $\begin{array}{l}\text { Etapa I } \\
\text { Pre lectura } \\
\text { Activar experiencias anteriores para acercarse al } \\
\text { texto. }\end{array}$ & $\begin{array}{l}\text { 1. Actividad - Grupo - profesor } \\
\text { Intercambio de opiniones sobre rutinas en general. } \\
\text { Aproximación } \\
\text { a las rutinas de aseo. } \\
\text { Destreza - Expresión oral interactiva } \\
\text { plurigestionada }\end{array}$ \\
\hline $\begin{array}{l}\text { Etapa II } \\
\text { Comprensión } \\
\text { Identificar elementos principales. Preparación. } \\
\text { Explorar el texto }\end{array}$ & $\begin{array}{l}\text { 1- Actividad - individual } \\
\text { Pareo-oraciones/figuras } \\
\text { Destreza } \\
\text { Comprensión lectora perceptiva } \\
\text { 2- Actividad - individual - parejas } \\
\text { Completar cuestionarios y comparar respuestas. } \\
\text { Destreza - Expresión escrita intensiva - } \\
\text { individual } \\
\text { Expresión oral auto gestionada - parejas }\end{array}$ \\
\hline
\end{tabular}




\begin{tabular}{|c|c|}
\hline Título > & ¡Cuestión de gustos! \\
\hline Estrategia $>$ & Modelo Interactivo: Etapa III \\
\hline Estrategia & Actividades y destrezas \\
\hline \multirow{3}{*}{$\begin{array}{l}\text { Etapa III } \\
\text { Interpretación } \\
\text { Identificar detalles } \\
\text { Se propone la lectura o el audio para lo } \\
\text { que cual se prepararon en las dos etapas } \\
\text { anteriores. }\end{array}$} & $\begin{array}{l}\text { 1. Actividad - individual } \\
\text { Obtener información del texto para lo cual se prepararon en } \\
\text { las dos etapas anteriores. } \\
\text { Destreza } \\
\text { Comprensión lectora selectiva/interactiva }\end{array}$ \\
\hline & $\begin{array}{l}\text { 2. Actividad - individual } \\
\text { Contestar cuestionarios } \\
\text { Destreza } \\
\text { Escritura intensiva }\end{array}$ \\
\hline & $\begin{array}{l}\text { 3. Actividad - grupo } \\
\text { Confirmar en un texto escrito la información oída para } \\
\text { conseguir información específica. } \\
\text { Destreza } \\
\text { Comprensión auditiva receptiva } \\
\text { Comprensión lectora selectiva } \\
\text { 4.Actividad - individual } \\
\text { Expresar opinión. Hablar apoyado en el texto. Contestar } \\
\text { preguntas formuladas por el compañero o profesor. } \\
\text { Destreza } \\
\text { Expresión oral de respuesta/intensiva/interactiva }\end{array}$ \\
\hline
\end{tabular}

\section{Bibliografía}

Carrel, P. (1993). Interactive text processing: implications for ESL/ second language reading classrooms. Por P. Carrel, J. Devine y D. E. Eske. (Eds.). Interactive approaches to second language reading. (239-259). Estados Unidos: Cambridge University Press.

Cassany, D., Luna, M. y Sanz, G. (2008). Enseñar lengua. Barcelona: GRAO.

Cros, E. (1997). El sujeto cultural sociocrítica y psicoanálisis. Argentina: Corregidor.

Eskey, D. (1993). Holding in the bottom: an interactive approach to the language problems of second language readers. Por P. Carrel, J. Devine y D. E. Eske. (Eds.). Interactive approaches to second language reading. (93-100). Estados Unidos: Cambridge University Press.

Eskey, D. y Grabe, W. (1993). Interactive models for second language reading: perspective on instruction. Por P. Carrel, J. Devine y D. E. Eske. (Eds.). Interactive approaches to second language reading. (223-238). Estados Unidos: Cambridge University Press.

Goodman, K. (1993). The reading process. Por P. Carrel, J. Devine y D. E. Eske. (Eds.) Interactive approaches to second language reading. (11-21). Estados Unidos: Cambridge University Press.

Krashen, S. (1981). Principles and Practice in Second Language. Englewood Cliffs, N.J.: Prentice- Hall. 
Lucchi-Riester, E. (2007). La actuación teatral y la adquisición de segunda lengua. (Tesis de Maestría). Indiana University. Estados Unidos. https://scholarworks.iupui.edu/ bitstream/handle/1805/998/Binder1.pdf;jsessionid=99D [Consulta 21 de enero de 2009].

Richards, J. (1990). The Language Teaching Matrix. England: Cambridge University Press.

Samuels, S. y Kamil, M. (1993). Models of the reading process. Por P. Carrel, J. Devine y D. E. Eske. (Eds.). Interactive approaches to second language reading. (22-36). Estados Unidos: Cambridge University Press.

Schnell, E. (2011). El Modelo Interactivo de Escucha y Lectura para la enseñanza del se en español. (Tesis de Maestría). Universidad de Costa Rica.

Shrum, J. y Glisan, E. (1994). Teacher's Handbook Contextualized language Instruction. Boston, Massachusetts: Heinle and Heinle Publishers.

Swaffar, J., Arens, K., y Byrnes, H. (1991). Redding for meaning. An integrated approach to language learning. Estados Unidos: Prentice Hall. 
